\title{
Application of the molecular interaction volume model to the assessment of thermodynamic properties of liquid Zn-Bi alloys
}

\author{
G.-H. Ding*, L.-M. Liang, P. Meng, L. Wang, Y.-X. Wang, Y.-J. Wang, R.-X. Gan
}

School of Mechanical and Vehicular Engineering, Bengbu University, Bengbu 233030, P. R. China

Received 9 May 2018, received in revised form 10 September 2018, accepted 18 September 2018

\begin{abstract}
This paper is an attempt to extract useful thermodynamic information from the experimental activity of zinc in Zn-Bi binary liquid alloy at different temperatures. The molecular interaction volume model (MIVM) was adopted to calculate a number of temperature dependent thermodynamic functions, including activity, free energy of mixing, concentration fluctuations in the long-wavelength limits, and diffusion. The reasonable agreement of the modeled thermodynamic parameters with the existing experimental data verified that the MIVM is quite convenient and reliable in the assessment of the thermodynamic properties of binary liquid alloys.
\end{abstract}

Ke y words: Zn-Bi, thermodynamic properties, molecular interaction volume model

\section{Introduction}

Most of the alloys are usually solidified from their liquid phases. Liquid alloys generate broad interest to researchers in materials, physics, and chemistry, since their atomic arrangements are significantly varied, which are heterocoordinated or homocoordinated. The liquid immiscible alloys had been experimentally investigated for 70 years. However, the complexity in the properties of mixing of binary liquid alloys is still less understood although some empirical and semiempirical models [1-5] have been proposed. Therefore, the study of the properties of binary liquid alloys demands extensive theoretical investigations at the microscopic level.

Elements $\mathrm{Bi}$ and $\mathrm{Zn}$ are the key compositions of the majority of multicomponent lead-free solders. The liquid binary system Bi-Zn separates forming Bi-rich and $\mathrm{Zn}$-rich phases within a certain range of temperatures and concentrations, which was experimentally evidenced by the temperature variation of physical properties such as viscosity, sound velocity, and thermodynamics [6-8]. The shape of the miscibility gap is remarkably flat and asymmetric, which enhanced difficulties during theoretical analysis [9]. There is some theoretical evidence from the points of quasi-lattice theory considering the combined effect of size ratio, entropic and enthalpic effect [10], and the perturbation approach that involves both energy of mixing and entropy of mixing [11].

In the study of multicomponent alloys, the MIVM is more popular, known for its reliability, its statistical thermodynamic origin and the fact that its application requires few parameters [12-15]. In this paper we have used the molecular interaction volume model, which is explained in the next sections, to predict the values of the thermodynamic and structural properties of $\mathrm{Zn}-\mathrm{Bi}$ liquid alloys at different temperatures.

\section{Molecular interaction volume model}

\subsection{Molar excess Gibbs energy of mixing and activity}

MIVM is based on the physical perspective of movement of liquid molecules which is known to be unlike that of solid or gas. The molecules of a liquid migrate non-randomly from one molecular cell to another unlike in gases and solids where molecules re-

*Corresponding author: e-mail address: ghding@chnu.edu.cn 
spectively move continuously in the irregular pattern and constantly vibrate at lattice sites $[16,17]$. From the nature of liquid molecular motion which allows each molecule to move into an adjacent hole as a central molecule, it is assumed that liquid molecules can move freely through a cell space, and the molecular interaction separation tending towards the cell diameter at certain temperature [18]. MIVM is built on the assumption that neighboring atoms are relative to each other, exchangeable, mobile and possess similar cells $[16,17]$.

Based on the non-random migration of liquid atoms, the MIVM is derived from statistical thermodynamics by Tao [12]. This model takes into account physical properties of the pure metals constituting the alloy, namely the molar volume, $V_{\mathrm{m}}$, and the first coordination number, $Z$, in the liquid state, which are temperature dependent $[19,20]$. The molar excess Gibbs energy $G^{\mathrm{E}}$ of the liquid mixture $i-j$ is given by

$$
\begin{aligned}
& G^{\mathrm{E}}=R T\left(x_{i} \ln \frac{V_{\mathrm{m} i}}{x_{i} V_{\mathrm{m} i}+x_{j} V_{\mathrm{m} j} B_{j i}}+\right. \\
& +x_{j} \ln \frac{V_{\mathrm{m} j}}{x_{j} V_{\mathrm{m} j}+x_{i} V_{\mathrm{m} i} B_{i j}}- \\
& \left.-\frac{x_{i} x_{j}}{2}\left(\frac{Z_{i} B_{j i} \ln B_{j i}}{x_{i}+x_{j} B_{j i}}+\frac{Z_{j} B_{i j} \ln B_{i j}}{x_{j}+x_{i} B_{i j}}\right)\right),
\end{aligned}
$$

where $R$ is the universal gas constant, $T$ is the temperature of the mixture, $x_{i}$ and $x_{j}$ are the molar fractions of $i$ and $j, B_{j i}$ and $B_{i j}$ are the pair-potential energy interaction parameters, which are defined as

$$
B_{i j}=\exp \left(-\frac{\varepsilon_{i j}-\varepsilon_{i i}}{k T}\right),
$$

where $k$ is the Boltzmann constant and $\varepsilon_{i j}$ is the pair potential energy of a central $j$ atom and its first nearest neighbor $i$.

Moreover, the activity of the components $i$ and $j$ can be expressed as

$$
\begin{aligned}
& \ln \alpha_{i}=\ln \frac{x_{i} V_{\mathrm{m} i}}{x_{i} V_{\mathrm{m} i}+x_{j} V_{\mathrm{m} j} B_{j i}}+ \\
& +x_{j}\left(\frac{V_{\mathrm{m} j} B_{j i}}{x_{i} V_{\mathrm{m} i}+x_{j} V_{\mathrm{m} j} B_{j i}}-\frac{V_{\mathrm{m} i} B_{i j}}{x_{j} V_{\mathrm{m} j}+x_{i} V_{\mathrm{m} i} B_{i j}}\right)- \\
& -\frac{x_{j}^{2}}{2}\left(\frac{Z_{i} B_{j i}^{2} \ln B_{j i}}{\left(x_{i}+x_{j} B_{j i}\right)^{2}}+\frac{Z_{j} B_{i j} \ln B_{i j}}{\left(x_{j}+x_{i} B_{i j}\right)^{2}}\right), \\
& \ln \alpha_{j}=\ln \frac{x_{j} V_{\mathrm{m} j}}{x_{j} V_{\mathrm{m} j}+x_{i} V_{\mathrm{m} i} B_{i j}}- \\
& -x_{i}\left(\frac{V_{\mathrm{m} j} B_{j i}}{x_{i} V_{\mathrm{m} i}+x_{j} V_{\mathrm{m} j} B_{j i}}-\frac{V_{\mathrm{m} i} B_{i j}}{x_{j} V_{\mathrm{m} j}+x_{i} V_{\mathrm{m} i} B_{i j}}\right)- \\
& -\frac{x_{i}^{2}}{2}\left(\frac{Z_{j} B_{i j}^{2} \ln B_{i j}}{\left(x_{j}+x_{i} B_{i j}\right)^{2}}+\frac{Z_{i} B_{j i} \ln B_{j i}}{\left(x_{i}+x_{j} B_{j i}\right)^{2}}\right) .
\end{aligned}
$$

To determine the value of $B_{j i}$ and $B_{i j}$, the activity of $i$ in binary alloy, $\alpha_{i}$, was fitted with Eq. (3) at a given temperature. The values of the physical parameters, $V_{\mathrm{m}}$ and $Z$, for the metals of interest in this work are taken from references [19] and [20]. The experimental activities were obtained by emf measurements in reference [21]. The final values of $B_{j i}$ and $B_{i j}$ are determined by minimizing the error err $=\sum_{i=1}^{n}\left|\alpha_{i, \exp }-\alpha_{i, \mathrm{MIVM}}\right| / n$, where $n$ is the number of experimental data points, as far as possible by adjusting $B_{j i}$ and $B_{i j}$.

The standard thermodynamic relation for the free energy of mixing $(G)$ is

$$
G=G^{\mathrm{E}}+G^{\mathrm{id}}
$$

with

$$
G^{\mathrm{id}}=R T\left(x_{i} \ln x_{i}+x_{j} \ln x_{j}\right),
$$

where $G^{\text {id }}$ is the molar ideal Gibbs energy.

\subsection{Concentration fluctuation}

The concentration fluctuation at the long wavelength limit $S_{x x}(0)$ is used to assess the response of demixing or ordering tendencies in the liquid alloy. It could serve as a sensitive test data of statistical models of liquid alloys, which can be easily obtained from standard relationship in terms of the free energy of mixing or in terms of activity, $\alpha_{i}$, as:

$$
\begin{aligned}
& S_{x x}(0)=R T\left(\frac{\partial^{2} G}{\partial x_{i}^{2}}\right)^{-1}=R T\left(\frac{\partial^{2} G}{\partial x_{j}^{2}}\right)^{-1}= \\
& =\alpha_{i}\left(1-x_{i}\right)\left(\frac{\partial \alpha_{i}}{\partial x_{i}}\right)^{-1}=\alpha_{j}\left(1-x_{j}\right)\left(\frac{\partial \alpha_{j}}{\partial x_{j}}\right)^{-1} .
\end{aligned}
$$

Using Eqs. (5) and (7), an expression to calculate $S_{x x}(0)$ is given by:

$$
S_{x x}(0)=\frac{x_{i} x_{j}}{1+x_{i} f\left(x_{i}, x_{j}\right)},
$$

where

$$
\begin{aligned}
& f\left(x_{i}, x_{j}\right)=\frac{V_{\mathrm{m} j} B_{j i}-V_{\mathrm{m} i}}{x_{i} V_{\mathrm{m} i}+x_{j} V_{\mathrm{m} j} B_{j i}}- \\
& -\frac{V_{\mathrm{m} i} V_{m j} B_{j i}}{\left(x_{i} V_{\mathrm{m} i}+x_{j} V_{\mathrm{m} j} B_{j i}\right)^{2}}+\frac{V_{\mathrm{m} i}^{2} B_{i j}^{2}}{\left(x_{j} V_{\mathrm{m} j}+x_{i} V_{\mathrm{m} i} B_{i j}\right)^{2}}+ \\
& +x_{j}\left(\frac{Z_{i} B_{j i}^{2} \ln B_{j i}}{\left(x_{i}+x_{j} B_{j i}\right)^{3}}+\frac{Z_{j} B_{i j}^{2} \ln B_{i j}}{\left(x_{j}+x_{i} B_{i j}\right)^{3}}\right)
\end{aligned}
$$

and for ideal mixing the molar excess Gibbs energy $G^{\mathrm{E}}$ given in Eq. (5) is equal to zero, and Eq. (8) reduces to

$$
S_{x x}^{\mathrm{id}}(0)=x_{i} x_{j}
$$




\subsection{Diffusivity}

The mixing behavior of two atomic species of a binary liquid alloy is required for a proper understanding of the dynamical properties such as diffusion at the microscopic level. Based on Darken's thermodynamic equation, the relation between diffusion and $S_{x x}(0)$ can be written as:

$$
\frac{D}{D_{\mathrm{id}}}=\frac{S_{x x}^{\mathrm{id}}(0)}{S_{x x}(0)},
$$

where $D$ is the mutual diffusion coefficient and $D_{\text {id }}$ is the intrinsic diffusion coefficient for the ideal mixture. The ratio $D / D_{\text {id }}$ indicates the mixing nature of molten alloys: $D / D_{\mathrm{id}}<1$ indicates the tendency of homocoordination while $D / D_{\text {id }}>1$ indicates the tendency of hetero-coordination and $D / D_{\text {id }}$ approaches 1 for ideal mixing.

\section{Results and discussion}

\subsection{Thermodynamic properties}

The activity is an important thermodynamic quantity which is a measure of the minimum Gibbs energy needed to convert $\mathrm{Zn}$ atom from its state in the $\mathrm{Zn}-\mathrm{Bi}$ solution to the pure state in any proposed extraction or refining process [18]. The experimental activities of zinc of $\mathrm{Zn}-\mathrm{Bi}$ liquid alloy were taken from the electromotive force study of Hayer et al. [21]. The theoretical model described in the preceding section has been used to study the concentration dependence of some thermodynamic quantities of $\mathrm{Zn}-\mathrm{Bi}$ binary liquid alloys at different temperatures. The values of $B_{j i}$ and $B_{i j}$ were determined by the overall fit of the experimental activity at different temperatures of the investigation, as given in Table 1 . It can be observed that the parameters reproduced fairly well the experimental values of activity and free energy of mixing at the indicated temperatures (Figs. 1 and 2). Djaballah et al. calculated the Gibbs energy of the liquid phase of the Zn-Bi alloy by using the Redlich-Kister model, considering the dependence of enthalpy and entropy of mixing of the liquid on the temperature [22]. Their results were compared with known experimental and our calculated results, as shown in Fig. 2. This good agreement between calculated and experimental values of these thermodynamic quantities justifies the use of this model for this study.

In Fig. 1, we observed that $\alpha_{\mathrm{Zn}}$ increases as $x_{\mathrm{Zn}}$ increases at each of the temperatures. However, if each of the $\alpha_{\mathrm{Zn}}$ isotherms is closely compared with others, we found that:

1. The positive departure from ideality of the activity values decreases with increasing temperature.
Table 1 . The values of $B_{j i}$ and $B_{i j}$ of $\mathrm{Zn}-\mathrm{Bi}$ alloy at different temperatures

\begin{tabular}{rcc}
\hline$T(\mathrm{~K})$ & $B_{j i}$ & $B_{i j}$ \\
\hline 873 & 1.1082 & 0.4456 \\
923 & 1.1234 & 0.4538 \\
973 & 1.1372 & 0.4612 \\
1023 & 1.1500 & 0.4676 \\
\hline
\end{tabular}

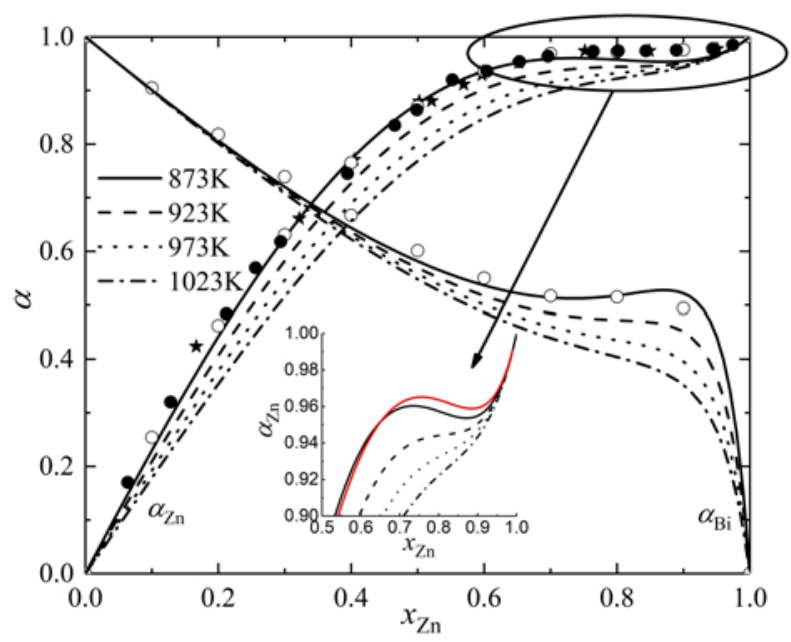

Fig. 1. Activity coefficients of ( $\alpha_{\mathrm{Zn}}$ and $\left.\alpha_{\mathrm{Bi}}\right)$ in $\mathrm{Zn}$-Bi liquid alloys computed via Eqs. (3) and (4) at different temperatures, respectively. $x_{\mathrm{Zn}}$ is the concentration of $\mathrm{Zn}$ in the liquid alloys. The open circles [28], bullets [21], and pentagrams [29] are the experimental data at $873 \mathrm{~K}$. The solid red line in the inset was calculated from the self-association model for $\gamma=2.874$ and $W / R T=1.326$ at $873 \mathrm{~K}$.

2. Throughout the entire composition of $\mathrm{Zn}$ in the alloy, $\alpha_{\mathrm{Zn}}$ at $1023 \mathrm{~K}$ is lower than at 873,923 , and $973 \mathrm{~K}$.

3 . The activity at $873 \mathrm{~K}$ has the maximum and minimum value at $x_{\mathrm{Zn}}=0.73$ and $x_{\mathrm{Zn}}=0.87$, respectively, as shown in the inset of Fig. 1. However, there is no extremum at 923,973 , and $1023 \mathrm{~K}$. The activity at $873 \mathrm{~K}$ was also calculated from the self-association model, which was proposed by Singh and Sommer [3], for $\gamma=2.874$ and $W / R T=1.326$. The extrema also appear at $x_{\mathrm{Zn}}=0.76$ and $x_{\mathrm{Zn}}=0.88$, respectively, as shown in Fig. 1. Similarly, the extremum is nonexistent at 923,973 , and $1023 \mathrm{~K}$ when the experimental activities were fitted by the self-association model.

Generally speaking, $\left|\frac{G}{R T}\right|$ increases as temperature increases at all concentrations, as shown in Fig. 2, which indicates that an increase in temperature enhances the atomic interactions in $\mathrm{Zn}-\mathrm{Bi}$ system. Moreover, the free energy of mixing is always asymmetric from 873 to $1023 \mathrm{~K}$. The position of the minimum 


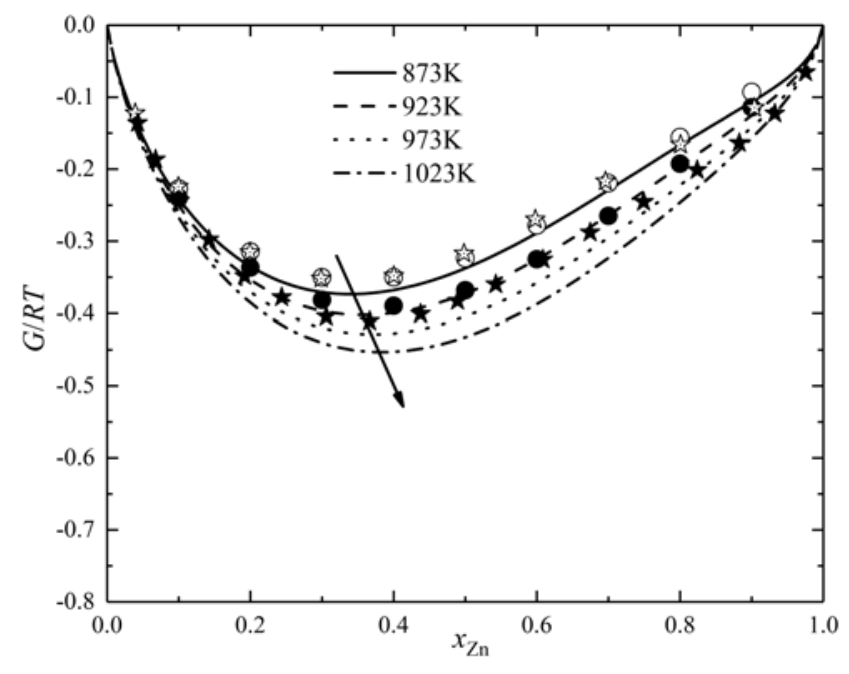

Fig. 2. Free energy of mixing: $G / R T$ versus $x_{\mathrm{Zn}}$ for $\mathrm{Zn}-\mathrm{Bi}$ liquid alloys at different temperatures, respectively. The open circles [28] and solid circles [21] are the experimental data at $873 \mathrm{~K}$ and $923 \mathrm{~K}$, respectively. The open pentagrams [28] and solid pentagrams [21] are the experimental data at 873 and $973 \mathrm{~K}$, respectively.

value of $G / R T$ shifts to $x_{\mathrm{Zn}}=0.50$ with increasing temperature, as shown by the arrow in Fig. 2, which suggests that the degree of segregation is weakened and the local order in the $\mathrm{Zn}-\mathrm{Bi}$ alloy moves to the hetero-coordination of unlike atoms as the temperature is increased considerably. In this range of composition, the temperature increase from 873 to $1023 \mathrm{~K}$ led to as much as $17.6 \%$ increase in $\left|\frac{G}{R T}\right|$. That at all temperatures the value of $\left|\frac{G}{R T}\right|$ is far less than 3.0 , is an indication that the energy of complex formation in $\mathrm{Zn}-\mathrm{Bi}$ liquid alloys is small and hence, $\mathrm{Zn}$ Bi liquid alloys are weakly interacting systems. Also, the asymmetric behavior relates to equiatomic composition typical of systems with atomic volume ratio $\frac{V_{\mathrm{m}, \mathrm{Bi}}}{V_{\mathrm{m}, \mathrm{Zn}}}>2.0[23]$ which in the present study is about 2.12 for $\mathrm{Zn}-$ Bi liquid alloy.

\subsection{Concentration fluctuation}

To understand the nature, strength, and level of influence of the order in the properties of the alloys, we consider the calculated $S_{x x}(0)$ using Eq. (8). Figure 3 shows a plot of $S_{x x}(0)$ against concentrations of zinc. The calculated values of $S_{x x}(0)$ are in agreement with the experimental values of $S_{x x}(0)$. The result can be used to understand the nature of atomic order in binary liquid alloys. Essentially, $S_{x x}(0)>S_{x x}^{\text {id }}(0)$ indicates the tendency of segregation in the liquid alloy. A perusal of Fig. 3 shows the demixing tendencies in $\mathrm{Zn}$ $\mathrm{Bi}$ liquid alloy at all the temperatures albeit at varying degrees. This is an indication that $\mathrm{Zn}-\mathrm{Bi}$ liquid alloys are homocoordinated and that the homocoordination

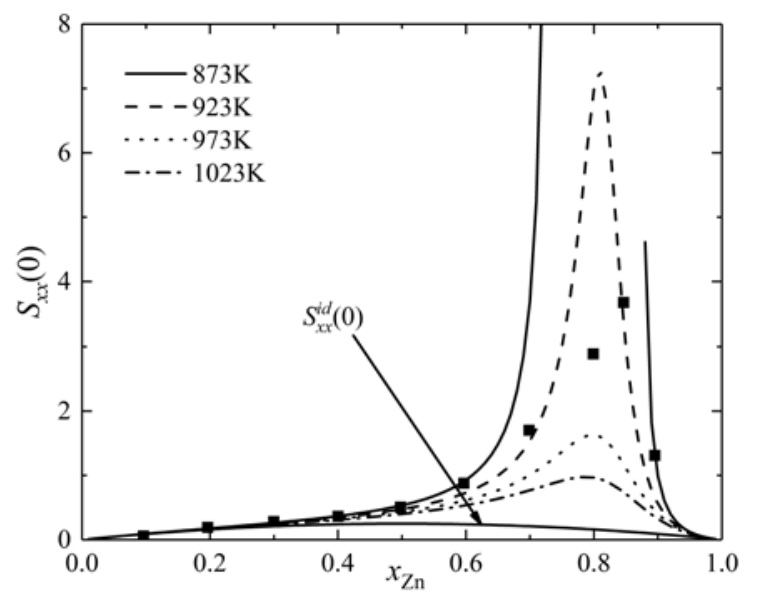

Fig. 3. $S_{x x}(0)$ in liquid Bi-Zn alloys at different temperatures. The square is the experimental data at $873 \mathrm{~K}[8]$.

which is strong is indicated by the relatively high peak at various temperatures when compared with weakly homocoordinated alloys like $\mathrm{Zn}{ }^{*} \mathrm{Cd}$ liquid alloys [24]. It should be noticed that there is a relatively large reduction in the peak value of $S_{x x}(0)$ as the temperature increased from 923 to $1023 \mathrm{~K}$. This trend in the reduction of the peak value of $S_{x x}(0)$ suggests some form of the reduction in the demixing tendencies of this alloy with an increase in temperature. This trend also suggests that with further increase in temperature, the $S_{x x}(0)$ of the alloy approaches ideal values. The reduction in the demixing tendencies of $\mathrm{Zn}-\mathrm{Bi}$ liquid alloy can be associated with the dissociation of the segregating clusters of the liquid alloy components. This dissociation of clusters occurs as a result of the increase in the temperature of the alloy.

\subsection{Diffusion}

It has been reported that transport properties such as diffusivities of metals in the liquid state are needed for many metallurgical processes [24]. Also, from the practical point of view, the viscosity is reported to be one of the key parameters for the design and optimization of metallurgical processes [25]. Gruner et al. in [26] reported that a study of the viscosity of liquid alloys contributes to the understanding of the relations between atomic structure and physical properties of alloys since properties like viscosity and diffusion are strongly sensitive to structural changes concerning temperature and composition. Hence, we have extended our investigation of the effects of temperature on the properties of $\mathrm{Zn}$-Bi liquid alloys, to investigate effects of temperature on its diffusivity properties, using Eq. (11). The results obtained are shown in Fig. 4. Although, in these figures due to the lack of experimental data, it is not possible to compare 


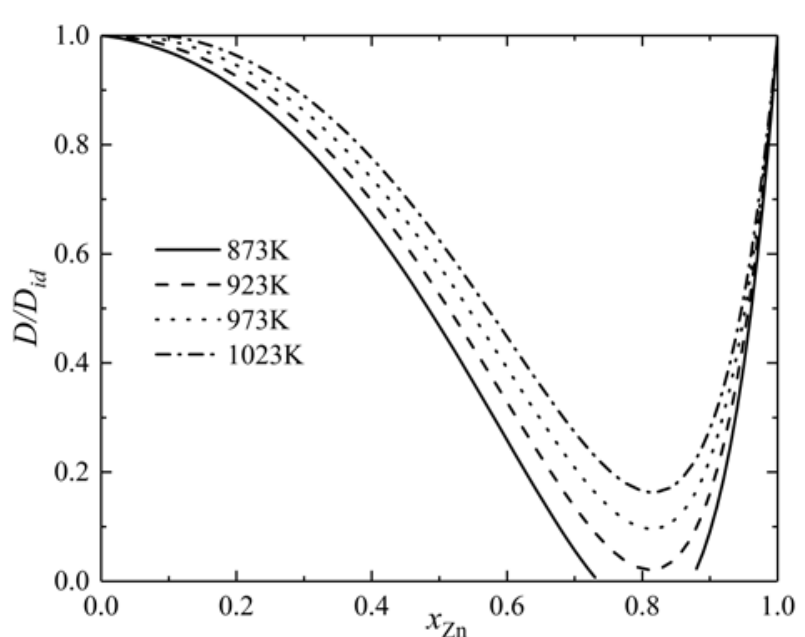

Fig. 4. Diffusion coefficients, $D / D_{\text {id }}$, of liquid Bi-Zn alloys at different temperatures.

computed and experimental results. Thus, these agreements serve as an evidence of the reliability of the following observations in Fig. 4:

1. At all temperatures, the magnitude of diffusivity reduces from unity towards minimum and then increases towards unity as the composition of $\mathrm{Zn}$ in the alloy increases;

2. The magnitude of the minimum diffusivity increases as the temperature increases;

3. Change in temperature resulted in increasing 6.74 fold in the magnitude of the diffusivity of $\mathrm{Zn}$ Bi liquid alloys (i.e., when $x_{\mathrm{Zn}}=0.81$, diffusivity $=$ 0.0211 at $923 \mathrm{~K}$, while diffusivity $=0.1633$ at $1023 \mathrm{~K}$ ).

In our analysis, the quantity of central importance is $S_{x x}(0)$ which is strongly concentration and temperature dependent for demixing liquid alloys. It is the temperature dependence of $S_{x x}(0)$ which allows us to investigate the temperature dependence of $D$. To exhibit the temperature dependence of diffusion coefficients, $\ln \left(D / D_{\text {id }}\right)$ and $D / D_{\text {id }}$ computed from Eq. (11) for the compositions $\mathrm{Zn}_{0.95} \mathrm{Bi}_{0.05}, \mathrm{Zn}_{0.70} \mathrm{Bi}_{0.30}$ and $\mathrm{Zn}_{0.30} \mathrm{Bi}_{0.70}$ are plotted in Fig. 5 as a function of $1 / T$. The results clearly suggest that $\ln \left(D / D_{\text {id }}\right)$ at $x_{\mathrm{Zn}}=0.7$, in the vicinity of the critical components is obviously in disagreement with the Arrhenius type of temperature dependence. However, far away from the critical composition, it displays a straight line behavior, i.e., at $x_{\mathrm{Zn}}=0.3$ and $x_{\mathrm{Zn}}=0.95$. It should be noted that $D / D_{\text {id }}$ for all the compositions depends linearly on $1 / T$, which is in agreement with the observations of Singh [27].

\section{Conclusions}

Molecular interaction volume model (MIVM) has been used to predict the thermodynamic activities of
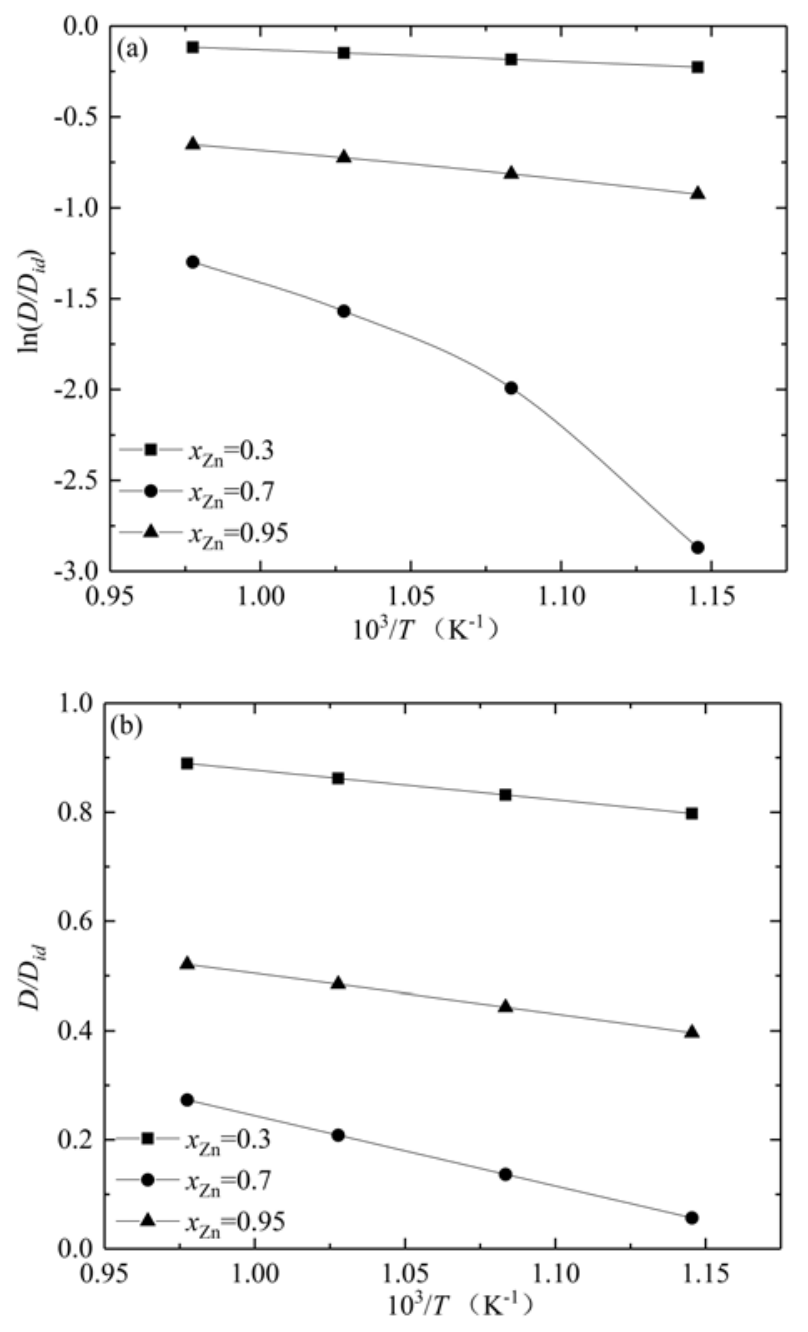

Fig. 5. Diffusion coefficients against temperature for the liquid $\mathrm{Zn}_{0.95} \mathrm{Bi}_{0.05}, \mathrm{Zn}_{0.70} \mathrm{Bi}_{0.30}, \mathrm{Zn}_{0.30} \mathrm{Bi}_{0.70}$, respectively: (a) $\left(\ln \left(D / D_{\text {id }}\right)\right.$ against $\left.T^{-1}\right)$ and (b) $\left(D / D_{\text {id }}\right.$ against $\left.T^{-1}\right)$.

all components in the liquid $\mathrm{Zn}-\mathrm{Bi}$ system in the temperature range $873-1023 \mathrm{~K}$. The results of our calculations reveal the following:

1. The calculated values of activity and free energy of mixing agree well with the experimental values at 873, 923, 973, and $1023 \mathrm{~K}$.

2. The calculated concentration fluctuation $S_{x x}(0)$ indicated the strong tendency of segregation in liquid $\mathrm{Zn}$-Bi alloys. With the temperature increasing, the homocoordinated clusters dissociated and the liquid alloy approached the ideal state.

3. The diffusivity properties increased quickly with the temperature increasing. $\ln \left(D / D_{\text {id }}\right)$ in the vicinity of the critical components is obviously in disagreement with the Arrhenius type of temperature dependence, while far away from the critical composition it displays a straight line behavior with $1 / T$. Moreover, $D / D_{\text {id }}$ depends linearly on $1 / T$ for all the compositions. 
The results obtained in this paper serve as the contribution to a complete thermodynamic description of Zn-Bi system. The results also confirm that MIVM is a reliable model for predicting the thermodynamic activities of liquid binary alloys.

\section{Acknowledgements}

This work was financially supported by the Key Project in the Youth Elite Support Plan in Universities of Anhui Province (Grant No. gxyqZD2018084) and Scientific Research Foundation for Talented Scholars of Bengbu University (Grant No. BBXY2018KYQD01).

\section{References}

[1] Axon, H. J.: Nature, 162, 1948, p. 997. doi:10.1038/162997a0

[2] Hildebrand, J. H., Scott, R. L.: The Solubility of NonElectrolytes. New York, Reinhold 1950.

[3] Singh, R. N., Sommer, F.: Rep. Prog. Phys., 60, 1997, p. 57. doi:10.1088/0034-4885/60/1/003

[4] Bhatia, A. B., Hargrove, W. H.: Phys. Rev. B, 10, 1974, p. 3186. doi:10.1103/PhysRevB.10.3186

[5] Bhatia, A. B., Singh, R. N.: Phys. Chem. Liq., 13, 1984, p. 177. doi:10.1080/00319108408080778

[6] Ueno, H., Kawakita, Y., Ohara, K., Tahara, S., Kohara, S., Itou, M., Takeda, S.: J. Phys.: Conf. Ser., 340, 2012, p. 012081. doi:10.1088/1742-6596/340/1/012081

[7] Mudry, S., Plevachuk, Y., Sklyarchuk, V., Yakymouych, A.: J. Non-Cryst. Solids, 354, 2008, p. 4415. doi:10.1016/j.jnoncrysol.2008.06.061

[8] Karlhuber, S., Mikula, A., Singh, R. N., Sommer, F.: J. Alloys Compd., 283, 1999, p. 198. doi:10.1016/S0925-8388(98)00887-1

[9] Malakhov, D. V.: Calphad, 24, 2000, p. 1. doi:10.1016/S0364-5916(00)00011-0

[10] Singh, R. N., Sommer, F.: Z. Metallkd., 83, 1992, p. 553.

[11] Kasem, M. R., Bhuiyan, G. M., Maruf, M. H. U.: J. Chem. Phys., 143, 2015, p. 034503. $\underline{\text { doi: } 10.1063 / 1.4926573}$
[12] Tao, D. P.: Thermochim. Acta, 363, 2000, p. 105. doi:10.1016/S0040-6031(00)00603-1

[13] Awe, O. E., Oshakuade, O. M.: Thermochim. Acta, 589, 2014, p. 47. doi:10.1016/i.tca.2014.05.009

[14] Poizeau, S., Sadoway, D. R.: J. Am. Chem. Soc., 135, 2013, p. 8260. doi:10.1021/ja4013886

[15] Kong, L. X., Yang, B., Xu, B. Q., Li, Y. F., Liu, D. C., Dai, Y. N.: Fluid Phase Equilib., 364, 2014, p. 1. doi:10.1016/i.fluid.2013.12.003

[16] Tao, D. P.: J. Alloys Compd., 457, 2008, p. 124. doi:10.1016/i.jallcom.2007.02.123

[17] Tao, D. P.: Metall. Mater. Trans. B, 32, 2001, p. 1205. doi:10.1007/s11663-001-0109-4

[18] Tomiska, J.: Thermochim. Acta, 314, 1998, p. 145. doi:10.1016/S0040-6031(97)00470-X

[19] Iida, T., Guthrie, R. I. L.: The Physical Properties of Liquid Metals. Oxford, Clarendon Press 1993.

[20] Tao, D. P.: Metall. Mater. Trans. A, 36, 2005, p. 3495. doi:10.1007/s11661-005-0023-5

[21] Hayer, E., Komarek, K. L., Mikula, A.: Monatsh. Chem., 107, 1976, p. 1437. doi:10.1007/BF01153922

[22] Djaballah, Y., Bennour, L., Bouharkat, F., BelgacemBouzida, A.: Modell. Simul. Mater. Sci. Eng., 13, 2005, p. 361. doi:10.1088/0965-0393/13/3/006

[23] Odusote, Y. A., Popoola, A. I., Oluyamo, S. S.: Appl. Phys. A, 122, 2016, p. 1. doi:10.1007/s00339-015-9591-4

[24] Awe, O. E., Azeez, A. A.: Appl. Phys. A, 123, 2017, p. 363. doi:10.1007/s00339-017-0977-3

[25] Yakymovych, A., Plevachuk, Y., Mudry, S., Brillo, J., Kobataka, H., Ipser, H.: Phys. Chem. Liq., 52, 2014, p. 562. doi:10.1080/00319104.2013.876639

[26] Gruner, S., Hoyer, W.: J. Alloys Compd., 460, 2008, p. 496. doi:10.1016/j.jallcom.2007.05.083

[27] Singh, R. N., Sommer, F.: Phys. Chem. Liq., 29, 1995, p. 191. doi:10.1080/00319109508028425

[28] Hultgren, R., Desai, P. D., Hawkins, D. T., Gleiser, M., Kelly, K. K.: Selected Values of the Thermodynamic Properties of Binary Alloys. Metals Park, ASM 1973.

[29] Kleppa, O. J.: J. Am. Chem. Soc., 74, 1952, p. 6052. doi:10.1021/ja01143a067 